\title{
MULTI-TYPE SUBCRITICAL BRANCHING PROCESSES IN A RANDOM ENVIRONMENT
}

\author{
BY VLADIMIR VATUTIN AND VITALI WACHTEL
}

\begin{abstract}
We study the asymptotic behavior of the survival probability of a multi-type branching process in a random environment. In the one-dimensional situation, the class of processes considered corresponds to the strongly subcritical case. We also prove a conditional limit theorem describing the distribution of the number of particles in the process given its survival for a long time.
\end{abstract}

Keywords: Branching process; random environment; survival probability; conditional limit theorem

2010 Mathematics Subject Classification: Primary 60J80

Secondary 60F99

\section{Introduction and statement of results}

In this paper we study the asymptotic properties of multi-type branching processes in random environments (BPREs). Informally speaking, such a process serves as a stochastic model for the evolution of a population with $p$ different types of particle, where each particle may have children of all types and the distribution of the number of descendants changes from generation to generation in some random fashion.

In order to give a rigorous definition of multi-type BPREs and to formulate our results, we must introduce some notation. First, $p$-dimensional deterministic vectors with nonnegative coordinates and $p \times p$ deterministic matrices with nonnegative entries are denoted by bold lower case symbols. In particular, $\mathbf{0}:=(0,0, \ldots, 0)$ and $\mathbf{1}:=(1,1, \ldots, 1)$. The standard basis vectors are denoted by $\boldsymbol{e}_{i}, i=1,2, \ldots, p$. For $p$-dimensional vectors $\boldsymbol{x}=\left(x^{1}, \ldots, x^{p}\right)$ and $\boldsymbol{y}=\left(y^{1}, \ldots, y^{p}\right)$, we set

$$
(\boldsymbol{x}, \boldsymbol{y}):=\sum_{i=1}^{p} x^{i} y^{i}, \quad|\boldsymbol{x}|:=\sum_{i=1}^{p}\left|x^{i}\right|, \quad \text { and } \quad \boldsymbol{x}^{\boldsymbol{y}}:=\prod_{i=1}^{p}\left(x^{i}\right)^{y^{i}} .
$$

For every $p$-tuple $\left(\mu^{1}, \mu^{2}, \ldots, \mu^{p}\right)$ of probability measures on $\mathbb{Z}_{+}^{p}$, define its multidimensional generating function $\boldsymbol{f}=\left(f^{1}, f^{2}, \ldots, f^{p}\right)$ by the relations

$$
f^{i}(s):=\sum_{z \in \mathbb{Z}_{+}^{p}} s^{z} \mu^{i}\{z\}, \quad s \in[0,1]^{p}, i=1,2, \ldots, p .
$$

We call any sequence $\left\{f_{n}, n \geq 1\right\}$ of multidimensional generating functions a varying environment, and interpret the corresponding measures $\mu_{n}^{i}$ as the offspring law for a particle of type $i$ in generation $n-1$. 
We now define a $p$-type branching process $Z_{n}=\left(Z_{n}^{1}, Z_{n}^{2}, \ldots, Z_{n}^{p}\right), n \geq 0$, where $Z_{n}^{i}$ is the number of type- $i$ particles in the process at moment $n$. This process has a deterministic starting point $\boldsymbol{Z}_{0}$ and the population sizes of the subsequent generations of the process are specified by the standard recursion

$$
Z_{n}^{i}=\sum_{j=1}^{p} \sum_{k=1}^{Z_{n-1}^{j}} X_{n, j, k}^{i}, \quad i=1,2, \ldots, p, n \geq 1,
$$

where $\boldsymbol{X}_{n, j, k}=\left(X_{n, j, k}^{1}, X_{n, j, k}^{2}, \ldots, X_{n, j, k}^{p}\right), k \geq 1$, are independent random vectors distributed according to $\mu_{n}^{j}$. Here and in what follows we denote random objects by upper case symbols using the respective bold symbols if the objects are vectors or matrices.

Equipping the set of all $p$-tuples $\left(\mu^{1}, \mu^{2}, \ldots, \mu^{p}\right)$ of probability measures with the total variation distance we obtain a metric space. Therefore, we may consider probability measures on this space. Because of the one-to-one correspondence between $p$-tuples of measures and $p$-dimensional generating functions, we can and do assume that we are given a probability measure on the space of generating functions. With this agreement in hand we call a sequence of independent, identically distributed (i.i.d.) random $p$-dimensional generating functions $\left\{\boldsymbol{F}_{n}=\right.$ $\left.\left(F_{n}^{1}, F_{n}^{2}, \ldots, F_{n}^{p}\right), n \geq 1\right\}$ a random environment. We say that $\left\{Z_{n}, n \geq 0\right\}$ is a $p$-type BPRE, if its conditional distribution is determined by (1) for every fixed realization of the environmental sequence.

Branching processes in a random environment with one type of particle have been intensively investigated during the last two decades and their properties are well understood and described in the literature. The reader can find a modern and unified presentation of the corresponding results in a recent book by Kersting and Vatutin [9]. The multidimensional case is much less studied and many basic questions, e.g. a detailed classification, asymptotics of the survival probability, and the corresponding conditional limit theorems, are still not answered in full generality. For instance, only recently an asymptotic representation for the survival probability in critical multi-type BPREs was found under relatively general conditions (see [10] and [11]).

The purpose of this note is to study asymptotic properties of a class of subcritical multi-type BPREs, which correspond to the so-called strongly subcritical BPREs with one type of particle.

It is a general phenomenon that the asymptotic behavior of BPREs is mainly specified by the properties of certain basic characteristics of the environment. In the case of multi-type processes the crucial role is played by the (random) mean matrices

$$
\boldsymbol{M}_{n}=\left(M_{n}^{i, j}\right)_{i, j=1}^{p}:=\left(\frac{\partial F_{n}^{i}}{\partial s^{j}}(\mathbf{1})\right)_{i, j=1}^{p}, \quad n \geq 1 .
$$

If the $\boldsymbol{F}_{n}$ are independent and distributed as a generating function $\boldsymbol{F}=\left(F^{1}, \ldots, F^{p}\right)$ then, obviously, the $\boldsymbol{M}_{n}$ are independent probabilistic copies of the random matrix

$$
\boldsymbol{M}=\left(M^{i, j}\right)_{i, j=1}^{p}:=\left(\frac{\partial F^{i}}{\partial s^{j}}(\mathbf{1})\right)_{i, j=1}^{p} .
$$

We assume that the distribution of $\boldsymbol{M}$ satisfies the following conditions.

(H0) $\mathbb{P}(\|\boldsymbol{M}\|>0)=1$, where $\|\boldsymbol{M}\|$ is the operator norm of $\boldsymbol{M}$.

(H1) The set $\Theta:=\left\{\theta>0: \mathbb{E}\left[\|\boldsymbol{M}\|^{\theta}\right]<\infty\right\}$ is nonempty. 
(H2) The support of the distribution of $\boldsymbol{M}$ acts strongly irreducibly on the semi-group of matrices with nonnegative entries, i.e. no proper finite union of subspaces of $\mathbb{R}^{p}$ is invariant with respect to all elements of the multiplicative semi-group generated by the support of $\boldsymbol{M}$.

(H3) There exists a positive number $\gamma>1$ such that

$$
1 \leq \frac{\max _{i, j} M^{i, j}}{\min _{i, j} M^{i, j}} \leq \gamma
$$

This last assumption is rather strong. Unfortunately, we do not know how to proceed without this restriction.

We also need to consider the so-called Hessian matrices

$$
\boldsymbol{B}(k):=\left(\frac{\partial^{2} F^{k}}{\partial s^{i} \partial s^{j}}(\mathbf{1})\right)_{i, j=1}^{p} \quad \text { and } \quad \boldsymbol{B}_{n}(k):=\left(\frac{\partial^{2} F_{n}^{k}}{\partial s^{i} \partial s^{j}}(\mathbf{1})\right)_{i, j=1}^{p}, \quad k=1,2, \ldots, p,
$$

and the random variables

$$
T:=\frac{1}{\|\boldsymbol{M}\|^{2}} \sum_{k=1}^{p}\|\boldsymbol{B}(k)\| \quad \text { and } \quad T_{n}:=\frac{1}{\left\|\boldsymbol{M}_{n}\right\|^{2}} \sum_{k=1}^{p}\left\|\boldsymbol{B}_{n}(k)\right\|, \quad n=1,2, \ldots
$$

Thus, $T_{n}$ are independent probabilistic copies of $T$. Along with conditions (H0)-(H3), we impose the following restriction on the distribution of $T$.

(H4) There exists $\varepsilon>0$ such that

$$
\mathbb{E}\left[\|\boldsymbol{M}\||\log T|^{1+\varepsilon}\right]<\infty .
$$

Using standard subadditivity arguments, we readily deduce that, for every $\theta \in \Theta$, the limit

$$
\lambda(\theta):=\lim _{n \rightarrow \infty}\left(\mathbb{E}\left[\left\|\boldsymbol{M}_{n} \cdots \boldsymbol{M}_{1}\right\|^{\theta}\right]\right)^{1 / n}<\infty
$$

is well defined. This function is an analogue of the moment generating function for the associated random walk in the case of BPREs with a single type of particle. Set

$$
\Lambda(\theta):=\log \lambda(\theta), \quad \theta \in \Theta .
$$

Theorem 1. Assume that conditions (HO)-(H4) hold, that the point $\theta=1$ belongs to the interior of the set $\Theta$, and that $\Lambda^{\prime}(1)<0$. Then

(a) there exists a vector $c=\left(c^{1}, \ldots, c^{p}\right)$ with strictly positive components such that

$$
\mathbb{P}\left(\left|\boldsymbol{Z}_{n}\right|>0 \mid \boldsymbol{Z}_{0}=\boldsymbol{e}_{i}\right) \sim c^{i} \lambda^{n}(1) \text { as } n \rightarrow \infty ;
$$

(b) for each $\boldsymbol{s} \in[0,1]^{p}, \boldsymbol{s} \neq \mathbf{1}$,

$$
\lim _{n \rightarrow \infty} \mathbb{E}\left[\boldsymbol{s}^{Z_{n}}|| Z_{n} \mid>0 ; Z_{0}=\boldsymbol{e}_{i}\right]=\Phi_{i}(\boldsymbol{s}),
$$

where $\Phi_{i}(s)$ is the probability generating function of a proper distribution on $\mathbb{Z}_{+}^{p}$ given at (10) below. 
If $p=1$ then the assumption $\Lambda^{\prime}(1)<0$ reduces to $\mathbb{E}[\boldsymbol{M} \log \boldsymbol{M}]<0$; one-type BPREs satisfying this condition are usually called strongly subcritical. Guivarch and Liu [7] studied the asymptotic behavior of the survival probability for one-type strongly subcritical processes; Geiger et al. [6] proved the corresponding conditional limit theorem for the distribution of the number of particles given survival. Dyakonova [3] obtained (2) under the assumption that all possible realizations of $\boldsymbol{M}$ have a common deterministic right eigenvector corresponding to the Perron-Frobenius root $\varrho(\boldsymbol{M})$ of $\boldsymbol{M}$. In this special case the condition $\Lambda^{\prime}(1)<0$ reduces to the inequality $\mathbb{E}[\varrho(\boldsymbol{M}) \log \varrho(\boldsymbol{M})]<0$. Dyakonova [4] studied the case $\mathbb{E}[\varrho(\boldsymbol{M}) \log \varrho(\boldsymbol{M})]=0$ under the assumption that there exists a common left eigenvector corresponding to the PerronFrobenius root of $\boldsymbol{M}$.

Our theorem and similar statements for strongly subcritical BPREs with one type of particle are very close to the classical results for subcritical Galton-Watson processes: for all these processes, there is purely geometric decay of the survival probability and a proper limit for the conditional distribution. The main difference between results for random and fixed environments consists in the fact that we cannot characterize the limit distribution in Theorem 1(b) by a functional equation.

\section{Proof of Theorem 1}

\subsection{Representation for the generating function of the process}

For every fixed realization of the environmental sequence $\boldsymbol{F}_{n}$ and $k<n$, we define

$$
\boldsymbol{F}_{k, n}(\boldsymbol{s}):=\boldsymbol{F}_{k+1} \circ \boldsymbol{F}_{k+2} \circ \cdots \circ \boldsymbol{F}_{n}(\boldsymbol{s}), \quad \boldsymbol{F}_{n, k}(\boldsymbol{s}):=\boldsymbol{F}_{n} \circ \boldsymbol{F}_{n-1} \circ \cdots \circ \boldsymbol{F}_{k+1}(\boldsymbol{s}),
$$

and set

$$
\boldsymbol{F}_{n, n}(\boldsymbol{s}):=\boldsymbol{s} .
$$

It is immediate from the definition of the process $\left\{\boldsymbol{Z}_{n}, n \geq 0\right\}$ that

$$
\mathbb{E}\left[\boldsymbol{s}^{\boldsymbol{Z}_{n}} \mid \boldsymbol{Z}_{0}=\boldsymbol{e}_{i}, \boldsymbol{F}_{1}, \boldsymbol{F}_{2}, \ldots, \boldsymbol{F}_{n}\right]=F_{0, n}^{i}(\boldsymbol{s}),
$$

where $\boldsymbol{F}_{0, n}(\boldsymbol{s}):=\left(F_{0, n}^{1}(\boldsymbol{s}), \ldots, F_{0, n}^{p}(\boldsymbol{s})\right)$. Therefore,

$$
\mathbb{E}\left[\boldsymbol{s}^{Z_{n}} \mid Z_{0}=\boldsymbol{e}_{i}\right]=\mathbb{E}\left[F_{0, n}^{i}(s)\right]
$$

Set $\boldsymbol{s}=\mathbf{0}$; then

$$
\mathbb{P}\left(\left|\boldsymbol{Z}_{n}\right|>0 \mid \boldsymbol{Z}_{0}=\boldsymbol{e}_{i}\right)=1-\mathbb{E}\left[F_{0, n}^{i}(\mathbf{0})\right]=\mathbb{E}\left[1-F_{0, n}^{i}(\mathbf{0})\right]
$$

Let $\boldsymbol{f}$ be the generating function of the $p$-tuple $\left(\mu^{1}, \mu^{2}, \ldots, \mu^{p}\right)$, and let $\boldsymbol{m}$ be the corresponding mean matrix, i.e.

$$
\boldsymbol{m}=\left(\frac{\partial f_{n}^{i}}{\partial s^{j}}(\mathbf{1})\right)_{i, j=1}^{p} .
$$

For any generating function $\boldsymbol{f}$ and matrix $\boldsymbol{a}$, define

$$
\psi_{f, a}(s):=\frac{|a|}{|a(1-f(s))|}-\frac{|a|}{|a m(1-s)|},
$$

where, with an abuse of notation, $|\cdot|$ here denotes the $L_{1}$-norm of matrices. 
Let $\boldsymbol{a}_{i}$ be the matrix with $a^{i, i}=1$ and $a^{k, l}=0$ for all $(k, l) \neq(i, i)$. Then, clearly,

$$
1-F_{0, n}^{i}(s)=\left|\boldsymbol{a}_{i}\left(\mathbf{1}-\boldsymbol{F}_{0, n}(\boldsymbol{s})\right)\right| \text {. }
$$

Using $\psi$, we can write

$$
\begin{aligned}
\frac{1}{1-F_{0, n}^{i}(s)} & =\frac{\left|\boldsymbol{a}_{i}\right|}{\left|\boldsymbol{a}_{i}\left(\mathbf{1}-\boldsymbol{F}_{0, n}(\boldsymbol{s})\right)\right|} \\
& =\frac{\left|\boldsymbol{a}_{i}\right|}{\left|\boldsymbol{a}_{i} \boldsymbol{M}_{1}\left(\mathbf{1}-\boldsymbol{F}_{1, n}(\boldsymbol{s})\right)\right|}+\psi_{\boldsymbol{F}_{1}, \boldsymbol{a}_{i}}\left(\boldsymbol{F}_{1, n}(\boldsymbol{s})\right) \\
& =\frac{1}{\left|\boldsymbol{a}_{i} \boldsymbol{M}_{1}\left(\mathbf{1}-\boldsymbol{F}_{1, n}(\boldsymbol{s})\right)\right|}+\psi_{\boldsymbol{F}_{1}, \boldsymbol{a}_{i}}\left(\boldsymbol{F}_{1, n}(\boldsymbol{s})\right) .
\end{aligned}
$$

Iterating this procedure yields

$$
\frac{1}{1-F_{0, n}^{i}(\boldsymbol{s})}=\frac{1}{\left|\boldsymbol{a}_{i} \boldsymbol{R}_{n}(\mathbf{1}-\boldsymbol{s})\right|}+\sum_{k=1}^{n} \frac{1}{\left|\boldsymbol{a}_{i} \boldsymbol{R}_{k}\right|} \psi_{\boldsymbol{F}_{k}, \boldsymbol{a}_{i} \boldsymbol{R}_{k-1}}\left(\boldsymbol{F}_{k, n}(\boldsymbol{s})\right),
$$

where $\boldsymbol{R}_{0}:=$ Id and

$$
\boldsymbol{R}_{k}:=\boldsymbol{M}_{1} \boldsymbol{M}_{2} \cdots \boldsymbol{M}_{k}, \quad k \geq 1 .
$$

Recall now that $\boldsymbol{F}_{k}$ are i.i.d. random elements, so we can substitute $\boldsymbol{F}_{k} \leftrightarrow \boldsymbol{F}_{n-k+1}$ and obtain

$$
\begin{aligned}
\mathbb{E}[1- & \left.F_{0, n}^{i}(\boldsymbol{s})\right] \\
& =\mathbb{E}\left[1-F_{n, 0}^{i}(\boldsymbol{s})\right] \\
& =\mathbb{E}\left[\left(\frac{1}{\left|\boldsymbol{a}_{i} \boldsymbol{L}_{n, 1}(\mathbf{1}-\boldsymbol{s})\right|}+\sum_{k=1}^{n} \frac{1}{\left|\boldsymbol{a}_{i} \boldsymbol{L}_{n, n-k+1}\right|} \psi_{\boldsymbol{F}_{n-k+1}, \boldsymbol{a}_{i} \boldsymbol{L}_{n, n-k+2}}\left(\boldsymbol{F}_{n-k, 0}(\boldsymbol{s})\right)\right)^{-1}\right] \\
& =\mathbb{E}\left[\left(\frac{1}{\left|\boldsymbol{a}_{i} \boldsymbol{L}_{n, 1}(\mathbf{1}-\boldsymbol{s})\right|}+\sum_{k=1}^{n} \frac{1}{\left|\boldsymbol{a}_{i} \boldsymbol{L}_{n, k}\right|} \psi_{\boldsymbol{F}_{k}, \boldsymbol{a}_{i} \boldsymbol{L}_{n, k+1}}\left(\boldsymbol{F}_{k-1,0}(\boldsymbol{s})\right)\right)^{-1}\right],
\end{aligned}
$$

where

$$
\boldsymbol{L}_{n, n+1}:=\mathbf{I d} \text { and } \boldsymbol{L}_{n, k}:=\boldsymbol{M}_{n} \boldsymbol{M}_{n-1} \cdots \boldsymbol{M}_{k}, \quad 1 \leq k \leq n .
$$

We show eventually that, as $n \rightarrow \infty$, the asymptotic behavior of $1-F_{0, n}^{i}(s)$ is determined by the summands corresponding to bounded values of $k$. To control $\psi_{\boldsymbol{F}_{k}, \boldsymbol{a}_{i} \boldsymbol{R}_{k}}$, we use Lemma 5 from [11]: under assumption (H3),

$$
0 \leq \psi_{\boldsymbol{F}_{k}, \boldsymbol{a}_{i} \boldsymbol{L}_{n, k+1}}\left(\boldsymbol{F}_{k-1,0}(\boldsymbol{s})\right) \leq \gamma p^{2} T_{k} \text { for all } \boldsymbol{s} \in[0,1]^{p},
$$

where we specify the value of $\psi_{\boldsymbol{F}_{k}, \boldsymbol{a}_{i} \boldsymbol{L}_{n, k+1}}(\boldsymbol{s})$ at $\boldsymbol{s}=\mathbf{1}$ by continuity.

\subsection{Exponential change of measure}

Define

$$
\mathbb{S}_{+}:=\left\{x \in \mathbb{R}_{+}^{p}:|x|=1\right\} .
$$

Then, for every matrix $\boldsymbol{m}$ with positive entries, the vector

$$
m \cdot x:=\frac{m x}{|m x|}
$$

is the projection of $\boldsymbol{m} \boldsymbol{x}$ on the set $\mathbb{S}_{+}$. 
Let $\mathcal{C}\left(\mathbb{S}_{+}\right)$denote the set of all continuous functions on $\mathbb{S}_{+}$. For $\theta \in \Theta, g \in \mathcal{C}\left(\mathbb{S}_{+}\right)$, and $\boldsymbol{x} \in \mathbb{S}_{+}$, define the operator

$$
P_{\theta} g(\boldsymbol{x}):=\mathbb{E}\left[|\boldsymbol{M} x|^{\theta} g(\boldsymbol{M} \cdot x)\right] .
$$

If conditions (H1)-(H3) hold then, according to Proposition 3.1 of $[1], \lambda(\theta)$ is the spectral radius of $P_{\theta}$ and there exist a unique strictly positive function $r_{\theta} \in \mathcal{C}\left(\mathbb{S}_{+}\right)$and a unique probability measure $l_{\theta}$ satisfying the scaling condition

$$
\int_{\mathbb{S}_{+}} r_{\theta}(\boldsymbol{x}) \mathrm{d} l_{\theta}(\boldsymbol{x})=1
$$

and such that

$$
l_{\theta} P_{\theta}=\lambda(\theta) l_{\theta}, \quad P_{\theta} r_{\theta}=\lambda(\theta) r_{\theta} .
$$

Following [2], introduce the functions

$$
p_{n}^{\theta}(\boldsymbol{x}, \boldsymbol{m}):=\frac{|\boldsymbol{m} x|^{\theta}}{\lambda^{n}(\theta)} \frac{r_{\theta}(\boldsymbol{m} \cdot x)}{r_{\theta}(\boldsymbol{x})}, \quad \boldsymbol{x} \in \mathbb{S}_{+} .
$$

It is easy to see that, for every $n \geq 1$, every $\boldsymbol{x} \in \mathbb{S}_{+}$, and every matrix $\boldsymbol{m}$,

$$
\mathbb{E}\left[p_{n+1}^{\theta}(\boldsymbol{x}, \boldsymbol{M m})\right]=p_{n}^{\theta}(\boldsymbol{x}, \boldsymbol{m})
$$

and, in particular,

$$
\mathbb{E}\left[p_{n}^{\theta}\left(\boldsymbol{x}, \boldsymbol{L}_{n, 1}\right)\right]=1 .
$$

For each $n \geq 1$, denote by $\mathcal{F}_{n}$ the $\sigma$-algebra generated by random elements $\boldsymbol{Z}_{1}, \boldsymbol{Z}_{2}, \ldots, \boldsymbol{Z}_{n}$ and $\boldsymbol{F}_{1}, \boldsymbol{F}_{2}, \ldots, \boldsymbol{F}_{n}$. Let $\mathbb{I}_{A}$ be the indicator of the event $A$. It follows from (7) that

$$
\mathbb{P}_{n}^{\theta}(A):=\mathbb{E}\left[p_{n}^{\theta}\left(\boldsymbol{x}, \boldsymbol{L}_{n, 1}\right) \mathbb{I}_{A}\right]
$$

is a probability measure on $\mathcal{F}_{n}$. Furthermore, (6) implies that $\left\{\mathbb{P}_{n}^{\theta}, n \geq 1\right\}$ is a sequence of consistent probability measures. It can be extended to a probability measure $\mathbb{P}^{\theta}$ on our original probability space $(\Omega, \mathcal{F})$.

We now take $\theta=1$ and apply the corresponding change of measure to representation (3). Since $1-F_{n, 0}^{i}(s)$ is measurable with respect to $\mathcal{F}_{n}$, we can write

$$
\begin{aligned}
\mathbb{E}\left[1-F_{n, 0}^{i}(\boldsymbol{s})\right] & =\lambda^{n}(1) r_{1}\left(\boldsymbol{e}_{i}\right) \mathbb{E}\left[p_{n}^{1}\left(\boldsymbol{e}_{i}, \boldsymbol{L}_{n, 1}\right) \frac{1-F_{n, 0}^{i}(\boldsymbol{s})}{\left|\boldsymbol{L}_{n, 1} \boldsymbol{e}_{i}\right| r_{1}\left(\boldsymbol{L}_{n, 1} \cdot \boldsymbol{e}_{i}\right)}\right] \\
& =\lambda^{n}(1) r_{1}\left(\boldsymbol{e}_{i}\right) \mathbb{E}^{1}\left[\frac{1-F_{n, 0}^{i}(\boldsymbol{s})}{\left|\boldsymbol{L}_{n, 1} \boldsymbol{e}_{i}\right| r_{1}\left(\boldsymbol{L}_{n, 1} \cdot \boldsymbol{e}_{i}\right)}\right] .
\end{aligned}
$$

Now apply (4), recall the definition of $\boldsymbol{a}_{i}$, and use the equality $\left|\boldsymbol{a}_{i} \boldsymbol{L}_{n, k}\right|=\left|\boldsymbol{e}_{i} \boldsymbol{L}_{n, k}\right|$. Then

$$
\mathbb{E}\left[1-F_{n, 0}^{i}(\boldsymbol{s})\right]=\lambda^{n}(1) r_{1}\left(\boldsymbol{e}_{i}\right) \mathbb{E}^{1}\left[\frac{1}{r_{1}\left(\boldsymbol{L}_{n, 1} \cdot \boldsymbol{e}_{i}\right)} \frac{\left|\boldsymbol{a}_{i} \boldsymbol{L}_{n, 1}(\mathbf{1}-\boldsymbol{s})\right|}{\left|\boldsymbol{L}_{n, 1} \boldsymbol{e}_{i}\right|} \boldsymbol{\Xi}_{n}(\boldsymbol{s})\right],
$$

where

$$
\Xi_{n}(\boldsymbol{s}):=\left(1+\sum_{k=1}^{n} \frac{\left|\boldsymbol{a}_{i} \boldsymbol{L}_{n, 1}(\mathbf{1}-\boldsymbol{s})\right|}{\left|\boldsymbol{e}_{i} \boldsymbol{L}_{n, k}\right|} \psi_{\boldsymbol{F}_{k}, \boldsymbol{a}_{i} \boldsymbol{L}_{n, k+1}}\left(\boldsymbol{F}_{k-1,0}(\boldsymbol{s})\right)\right)^{-1}
$$


For brevity, write

$$
\tilde{\psi}_{n, k}(\boldsymbol{s}):=\psi_{\boldsymbol{F}_{k}, \boldsymbol{a}_{i} \boldsymbol{L}_{n, k+1}}\left(\boldsymbol{F}_{k-1,0}(\boldsymbol{s})\right) .
$$

Fix some $N \geq 1$; we study the asymptotic behavior of the expectation

$$
K_{n}(N ; \boldsymbol{s}):=\mathbb{E}^{1}\left[\frac{1}{r_{1}\left(\boldsymbol{L}_{n, 1} \cdot \boldsymbol{e}_{i}\right)} \frac{\left|\boldsymbol{a}_{i} \boldsymbol{L}_{n, 1}(\mathbf{1}-\boldsymbol{s})\right|}{\left|\boldsymbol{L}_{n, 1} \boldsymbol{e}_{i}\right|} \Xi_{n, N}(\boldsymbol{s})\right],
$$

where

$$
\boldsymbol{\Xi}_{n, N}(\boldsymbol{s}):=\left(1+\sum_{k=1}^{N} \frac{\left|\boldsymbol{a}_{i} \boldsymbol{L}_{n, 1}(\mathbf{1}-\boldsymbol{s})\right|}{\left|\boldsymbol{e}_{i} \boldsymbol{L}_{n, k}\right|} \tilde{\psi}_{n, k}(\boldsymbol{s})\right)^{-1}
$$

For each $k \leq N$,

$$
\frac{\left|\boldsymbol{a}_{i} \boldsymbol{L}_{n, 1}(\mathbf{1}-\boldsymbol{s})\right|}{\left|\boldsymbol{e}_{i} \boldsymbol{L}_{n, k}\right|}=\frac{\left|\boldsymbol{a}_{i} \boldsymbol{L}_{n, N} \boldsymbol{L}_{N-1,1}(\mathbf{1}-\boldsymbol{s})\right|}{\left|\boldsymbol{e}_{i} \boldsymbol{L}_{n, N} \boldsymbol{L}_{N-1, k}\right|} .
$$

By Theorem 1 of [8], there exist sequences of random numbers $\left\{\lambda_{n}(N)>0, n \geq 1\right\}$ and random vectors $\left\{\boldsymbol{U}_{n}^{(N)}, \boldsymbol{V}_{n}^{(N)}, n \geq 1\right\}$ such that, as $n \rightarrow \infty$,

$$
\frac{\boldsymbol{L}_{n, N}}{\lambda_{n}(N)}-\boldsymbol{U}_{n}^{(N)} \otimes \boldsymbol{V}_{n}^{(N)} \rightarrow 0 \quad \text { almost surely (a.s.). }
$$

(Here and in what follows we agree to associate with vectors $\boldsymbol{u}=\left(u^{1}, \ldots, u^{p}\right)$ and $\boldsymbol{v}=$ $\left(v^{1}, \ldots, v^{p}\right)$ the matrix $\boldsymbol{u} \otimes \boldsymbol{v}=\left(u_{i} v_{j}\right)_{i, j=1}^{p}$.) Besides, as $n \rightarrow \infty$, the sequence of random vectors $\left\{\left(\boldsymbol{U}_{n}^{(N)}, \boldsymbol{V}_{n}^{(N)} /\left|\boldsymbol{V}_{n}^{(N)}\right|\right), n \geq 1\right\}$ converges weakly to a vector $\left(\boldsymbol{U}^{(N)}, \boldsymbol{V}^{(N)}\right)$. Consequently, as $n \rightarrow \infty$, the sequence of ratios

$$
\frac{\left|\boldsymbol{a}_{i} \boldsymbol{L}_{n, N} \boldsymbol{L}_{N-1,1}(\mathbf{1}-\boldsymbol{s})\right|}{\left|\boldsymbol{e}_{i} \boldsymbol{L}_{n, N} \boldsymbol{L}_{N-1, k}\right|}=\frac{\left|\boldsymbol{a}_{i} \boldsymbol{L}_{n, N} \boldsymbol{L}_{N-1,1}(\mathbf{1}-\boldsymbol{s}) /\left(\lambda_{n}(N)\left|\boldsymbol{V}_{n}^{(N)}\right|\right)\right|}{\left|\boldsymbol{e}_{i} \boldsymbol{L}_{n, N} \boldsymbol{L}_{N-1, k} /\left(\lambda_{n}(N)\left|\boldsymbol{V}_{n}^{(N)}\right|\right)\right|}, \quad k \leq N,
$$

converges weakly to

$$
\frac{\left|\boldsymbol{a}_{i}\left(\boldsymbol{U}^{(N)} \otimes \boldsymbol{V}^{(N)}\right) \boldsymbol{L}_{N-1,1}(\mathbf{1}-\boldsymbol{s})\right|}{\left|\boldsymbol{e}_{i}\left(\boldsymbol{U}^{(N)} \otimes \boldsymbol{V}^{(N)}\right) \boldsymbol{L}_{N-1, k}\right|}, \quad k \leq N .
$$

By the same arguments, as $n \rightarrow \infty$, the vectors $\left(\tilde{\psi}_{n, 1}^{(N)}(s), \ldots, \tilde{\psi}_{n, N}^{(N)}(s)\right)$ converge weakly to a vector $\left(\tilde{\psi}_{1}^{(N)}(\boldsymbol{s}), \ldots, \tilde{\psi}_{N}^{(N)}(\boldsymbol{s})\right)$, and $r_{1}\left(\boldsymbol{L}_{n, 1} \cdot \boldsymbol{e}_{i}\right)$ converges weakly to a bounded random variable. Therefore, there exists

$$
\lim _{n \rightarrow \infty} K_{n}(N ; s)=: K^{i}(N ; s)
$$

and the sequence $K^{i}(N ; s)$ is decreasing in $N$ for each fixed $s=\left(s^{1}, \ldots, s^{p}\right)$.

Let $A(\boldsymbol{s})=\left\{1 \leq j \leq p: s^{j}<1\right\}$. Setting $\Delta(s):=\min _{j \in A(s)}\left(1-s^{j}\right)$ and using Lemma 2 of [5], it is not difficult to check that

$$
\frac{\Delta(\boldsymbol{s})}{p^{2} \gamma^{2}} \leq \frac{\left|\boldsymbol{a}_{i} \boldsymbol{L}_{n, 1}(\mathbf{1}-\boldsymbol{s})\right|}{\left|\boldsymbol{L}_{n, 1} \boldsymbol{e}_{i}\right|} \leq \frac{\left|\boldsymbol{e}_{i} \boldsymbol{L}_{n, 1}\right|}{\left|\boldsymbol{L}_{n, 1} \boldsymbol{e}_{i}\right|} \leq \gamma^{2} p^{2}, \quad n \geq 1 .
$$

Combining these estimates with the fact that $r_{1}$ is bounded away from 0 , for some absolute constant $C=C(s)$, we obtain the estimates

$$
\begin{aligned}
0 & \leq K_{n}(N ; \boldsymbol{s})-\mathbb{E}^{1}\left[\frac{1}{r_{1}\left(\boldsymbol{L}_{n, 1} \cdot \boldsymbol{e}_{i}\right)} \frac{\left|\boldsymbol{a}_{i} \boldsymbol{L}_{n, 1}(\mathbf{1}-\boldsymbol{s})\right|}{\left|\boldsymbol{L}_{n, 1} \boldsymbol{e}_{i}\right|} \Xi_{n}(\boldsymbol{s})\right] \\
& \leq C \mathbb{E}^{1}\left[\frac{\sum_{k=N+1}^{n} \tilde{\psi}_{k}(\boldsymbol{s})\left|\boldsymbol{a}_{i} \boldsymbol{L}_{n, 1}(\mathbf{1}-\boldsymbol{s})\right| /\left|\boldsymbol{e}_{i} \boldsymbol{L}_{n, k}\right|}{1+\sum_{k=N+1}^{n} \tilde{\psi}_{k}(\boldsymbol{s})\left|\boldsymbol{a}_{i} \boldsymbol{L}_{n, 1}(\mathbf{1}-\boldsymbol{s})\right| /\left|\boldsymbol{e}_{i} \boldsymbol{L}_{n, k}\right|}\right] .
\end{aligned}
$$


Clearly,

$$
\left|\boldsymbol{a}_{i} \boldsymbol{L}_{n, 1}(\mathbf{1}-\boldsymbol{s})\right|=\left|\boldsymbol{a}_{i} \boldsymbol{L}_{n, k} \boldsymbol{L}_{k-1,1}(\mathbf{1}-\boldsymbol{s})\right| \leq\left|\boldsymbol{e}_{i} \boldsymbol{L}_{n, k}\right|\left\|\boldsymbol{L}_{k-1,1}\right\|, \quad k \leq n .
$$

From this bound and (5), for some constant $C_{1}$, we obtain

$$
\begin{aligned}
0 & \leq K_{n}(N ; \boldsymbol{s})-\mathbb{E}^{1}\left[\frac{1}{r_{1}\left(\boldsymbol{L}_{n, 1} \cdot \boldsymbol{e}_{i}\right)} \frac{\left|\boldsymbol{a}_{i} \boldsymbol{L}_{n, 1}(\mathbf{1}-\boldsymbol{s})\right|}{\left|\boldsymbol{L}_{n, 1} \boldsymbol{e}_{i}\right|} \Xi_{n}(\boldsymbol{s})\right] \\
& \leq C_{1} \mathbb{E}^{1}\left[\frac{\sum_{k=N+1}^{\infty}\left\|\boldsymbol{L}_{k-1,1}\right\| T_{k}}{1+\sum_{k=N+1}^{\infty}\left\|\boldsymbol{L}_{k-1,1}\right\| T_{k}}\right]:=K(N) .
\end{aligned}
$$

Suppose that we can show that the series

$$
\Psi:=1+\sum_{k=1}^{\infty}\left\|\boldsymbol{L}_{k-1,1}\right\| T_{k}<\infty \quad \mathbb{P}^{1} \text {-a.s. }
$$

Then we should have

$$
K(N) \rightarrow 0 \quad \text { as } N \rightarrow \infty .
$$

Combining this with (8) also implies the existence of the limit

$$
\lim _{n \rightarrow \infty} \mathbb{E}^{1}\left[\frac{1}{r_{1}\left(\boldsymbol{L}_{n, 1} \cdot \boldsymbol{e}_{i}\right)} \frac{\left|\boldsymbol{a}_{i} \boldsymbol{L}_{n, 1}(\mathbf{1}-\boldsymbol{s})\right|}{\left|\boldsymbol{L}_{n, 1} \boldsymbol{e}_{i}\right|} \Xi_{n}(\boldsymbol{s})\right]=\lim _{N \rightarrow \infty} K^{i}(N ; \boldsymbol{s})=: \phi_{i}(\boldsymbol{s}) .
$$

But the limit on the right-hand side exists, because the sequence $K^{i}(N ; s)$ is decreasing in $N$. Taking into account (9) and the estimate $\Xi_{n}(s) \geq \Psi^{-1}$, we conclude that this limit is strictly positive for each $s \in[0,1]^{p}, \boldsymbol{s} \neq \mathbf{1}$. In addition, $\phi_{i}(\mathbf{1})=0$, since $\Xi_{n}(s) \leq 1$.

With these results to hand we deduce that, as $n \rightarrow \infty$,

$$
\mathbb{P}\left(\left|\boldsymbol{Z}_{n}\right|>0 \mid \boldsymbol{Z}_{0}=\boldsymbol{e}_{i}\right)=\mathbb{E}\left[1-F_{0, n}^{i}(\mathbf{0})\right] \sim \lambda^{n}(1) r_{1}\left(\boldsymbol{e}_{i}\right) \phi_{i}(\mathbf{0})
$$

and

$$
\lim _{n \rightarrow \infty} \mathbb{E}\left[\boldsymbol{s}^{\boldsymbol{Z}_{n}}|| \boldsymbol{Z}_{n} \mid>0 ; \boldsymbol{Z}_{0}=\boldsymbol{e}_{i}\right]=1-\lim _{n \rightarrow \infty} \frac{\mathbb{E}\left[1-F_{0, n}^{i}(\boldsymbol{s})\right]}{\mathbb{E}\left[1-F_{0, n}^{i}(\mathbf{0})\right]}=1-\frac{\phi_{i}(\boldsymbol{s})}{\phi_{i}(\mathbf{0})}=: \Phi_{i}(\boldsymbol{s}) .
$$

To complete the proof of the theorem, it remains to check that (9) holds.

Note first that our assumption that the point 1 belongs to the interior of $\Theta$ proves the finiteness of $\mathbb{E}^{1}[\log \|\boldsymbol{M}\|]$. Moreover, by condition (H3),

$$
\min _{x \in \mathbb{S}_{+}} \frac{|x \boldsymbol{M}|}{|x|} \geq \gamma^{-1}\|\boldsymbol{M}\|
$$

Then all the conditions of Theorem 2 of [8] are satisfied and, therefore,

$$
\lim _{k \rightarrow \infty} \frac{\log \left\|L_{k, 1}\right\|}{k}=\Lambda^{\prime}(1)<0 \quad \mathbb{P}^{1} \text {-a.s. }
$$

In particular, for every $\varepsilon>0$,

$$
\left\|L_{k, 1}\right\|=O\left(\mathrm{e}^{-k^{1-\varepsilon / 2}}\right) \quad \mathbb{P}^{1} \text {-a.s. }
$$


By Markov's inequality,

$$
\mathbb{P}^{1}\left(\log T_{k}>k^{(1+\delta) /(1+\varepsilon)}\right) \leq \frac{\mathbb{E}^{1}\left[\left|\log T_{k}\right|^{1+\varepsilon}\right]}{k^{1+\delta}} .
$$

Now using the definition of the measure $\mathbb{P}^{1}$, we obtain

$$
\mathbb{P}^{1}\left(\log T_{k}>k^{(1+\delta) /(1+\varepsilon)}\right) \leq C_{2} \frac{\mathbb{E}\left[\|\boldsymbol{M}\||\log T|^{1+\varepsilon}\right]}{k^{1+\delta}}
$$

for some constant $C_{2}$. Taking into account condition (H4) and applying the Borel-Cantelli lemma, we conclude that

$$
T_{k}=O\left(\mathrm{e}^{k^{(1+\delta) /(1+\varepsilon)}}\right) \quad \mathbb{P}^{1} \text {-a.s. }
$$

Combining this estimate with (11) and choosing sufficiently small $\delta$, we obtain (9).

\section{Acknowledgements}

This work was supported by the Russian Science Foundation under the grant 17-11-01173 and was carried out at Novosibirsk State University.

\section{References}

[1] Buraczewski, D., Damek, E., Guivarc'h, Y. and Mentemeier, S. (2014). On multidimensional Mandelbrot cascades. J. Difference Equat. Appl. 20, 1523-1567.

[2] Collamore, J. F. And Mentemeier, S. (2016). Large excursions and conditioned laws for recursive sequences generated by random matrices. Preprint. Available at https://arxiv.org/abs/1608.05175v1.

[3] Dyakonova, E. (2008). On subcritical multi-type branching process in random environment. In Proc. 5th Colloquium on Mathematics and Computer Science (Discrete Math. Theoret. Comput. Sci. AI), Association of Discrete Mathematics and Theoretical Computer Science, Nancy, pp. 397-404.

[4] Dyakonova, E. E. (2013). Multitype subcritical branching processes in a random environment. Proc. Steklov Inst. Math. 282, 80-89.

[5] Furstenberg, H. and Kesten, H. (1960). Products of random matrices. Ann. Math. Statist. 31, 457-469.

[6] Geiger, J., Kersting, G. And Vatutin, V. A. (2003). Limit theorems for subcritical branching processes in random environment. Ann. Inst. H. Poincaré Prob. Statist. 39, 593-620.

[7] Guivarc'H, Y. AND LiU, Q. (2001). Asymptotic properties of branching processes in random environment. C. R. Acad. Sci. Paris 332, 339-344.

[8] Hennion, H. (1997). Limit theorems for products of positive random matrices. Ann. Prob. 25, 1545-1587.

[9] Kersting, G. and Vatutin, V. (2017). Discrete Time Branching Processes in Random Environment. ISTE, London.

[10] Le Page, E., Peigné, M. and Pham, C. (2016). The survival probability of a critical multi-type branching process in i.i.d. random environment. Preprint. Available at https://hal.archives-ouvertes.fr/hal-01376091v1.

[11] VATUtin, V. A. AND D'Yakonova, E. E. (2017). Multitype branching processes in random environment: survival probability for the critical case. Teor. Veroyat. Primen. 62, 634-653 (in Russian).

\section{VLADIMIR VATUTIN, Novosibirsk State University and Steklov Mathematical Institute}

Steklov Mathematical Institute, Gubkin Street 8, 119991 Moscow, Russia. Email address: vatutin@mi.ras.ru

\section{VITALI WACHTEL, Universität Augsburg}

Institut für Mathematik, Universität Augsburg, 86135 Augsburg, Germany.

Email address: vitali.wachtel@mathematik.uni-augsburg.de 